E-009 INITIAL EXPERIENCE OF CURATIVE TRANSVENOUS EMBOLIZATION OF CEREBRAL ARTERIOVENOUS MALFORMATIONS USING RAPID VENTRICULAR PACING FOR FLOW ARREST

${ }^{1}$ A White, '1 Corona, ${ }^{2} R$ Joshi, ${ }^{3} B$ Welch*. ${ }^{1}$ Radiology, UT Southwestern, Dallas, TX; ${ }^{2}$ Anesthesiology and Pain Management, UT Southwestern, Dallas, TX; ${ }^{3}$ Neurological Surgery, UT Southwestern, Dallas, TX

\subsection{6/neurintsurg-2021-SNIS.105}

Introduction/Purpose Endovascular embolization of cerebral arteriovenous malformations (AVMs) has been traditionally used as part of a multimodal treatment paradigm in combination with radiosurgery and/or microsurgical resection, with embolization most commonly occurring via a transarterial route. In recent years, transvenous embolization has been proposed as an alternative to this paradigm and has been shown to be safe, effective, and curative in $>90 \%$ of cases. Curative embolization via the transvenous route often necessitates some means of arterial inflow control. Rapid ventricular pacing (RVP) is a technique for intraoperative flow reduction that has been successfully used by Cardiologists and Neurosurgeons for over a decade, though published reports in the Neurointerventional literature have been sparse. To contribute to this gap, we present our initial experience of curative transvenous embolization of AVMs aided by flow arrest with RVP.

Materials and Methods A total of four consecutive patients over a 4-month period were included in this study. All patients were female, ranged in age from 18 to 65, and all had experienced prior rupture of their AVM. All AVMs were Spetzler-Martin (SM) grade III and were located in the left thalamus, left midbrain $(n=2)$, and right frontal lobe. Three of $4 \mathrm{AVMs}$ had a single draining vein. Following induction of general anesthesia, an arterial line and right ventricular pacing catheter were placed. Physiologic parameters recorded intra-operatively include mean arterial pressure (MAP) and end-tidal $\mathrm{CO}_{2}\left(\mathrm{EtCO}_{2}\right)$. RVP was performed over a period of 2-4 minutes (mean: $3.2 \mathrm{~min}$ ) at a rate of 182 beats per minute, during which time Onyx (Medtronic, Irvine, CA) was injected.

Results RVP produced an immediate and sustained MAP reduction of $37.8 \mathrm{mmHg}$, and mean $\mathrm{EtCO}_{2}$ during this period was $9.2 \mathrm{mmHg}$. Transvenous partial coiling of the draining vein prior to Onyx delivery was performed in 2 patients in order to facilitate deeper penetration into a remotely located nidus. A single patient required a second round of RVP for completion of Onyx injection. A curative result was achieved in all patients with a single draining vein, all of which were stable on 3 month follow up angiography. A small residual nidus with persistent, albeit greatly reduced, arteriovenous shunting occurred in the one patient with multiple draining veins. There was 1 procedural complication (inadvertent transarterial embolization resulting in a thalamic stroke) and 1 complication related to RVP (development of polymorphic ventricular tachycardia after RVP necessitating defibrillation $\mathrm{x} 5$ and several rounds of cardiopulmonary resuscitation prior to spontaneous recovery). No delayed or hemorrhagic complications were encountered.

Conclusion RVP may be successfully used to obtain flow arrest during neuroendovascular procedures, particularly those that require precise and controllable delivery of embolic material.
This is a highly effective technique for curative transvenous embolization of cerebral AVMs in carefully selected patients, particularly those with AVMs that are small, with a single draining vein, and unresectable due to deep location (SM grade III or less). These preliminary results highlight the need for future studies to validate efficacy and optimize patient selection.

Disclosures A. White: None. J. Corona: None. R. Joshi: None. B. Welch: 2; C; Medtronic, Stryker, Microvention. 6; C; Peter Lazic Inc.

\section{E-010 STRICT ASPECTS CUTOFFS IN ENDOVASCULAR THERAPY SELECTION PARADIGMS MAY HARM PATIENT OUTCOMES}

${ }^{1} \mathrm{R}$ Abdelkhaleq*, ${ }^{2} \mathrm{C}$ De la Garza, 'Y Kim, 'S Khose, 'S Salazar Marioni, 'S Sheth. ${ }^{1}$ Neurology, McGovern Medical School, Houston, TX; ${ }^{2}$ Neurology, Baylor College of Medicine, Houston, $T X$

\subsection{6/neurintsurg-2021-SNIS.106}

Introduction With the results of the 2015 and 2018 randomized clinical trials, hospital-based protocols and society-based guidelines have emerged to define candidacy for endovascular stroke therapy (EST) for large vessel occlusion (LVO) acute ischemic stroke. ASPECT score cutoffs, typically $\geq 5$, are frequently used, in keeping with trial results. On the other hand, the likelihood of benefit to patients may not drop off substantially at that cutoff value. Here, we assess the frequency of good outcomes in patients across the full range of ASPECTS, and then measure the effect of applying strict cutoffs to EST eligibility based on ASPECTS $\geq 5$.

Methods From our prospectively maintained observational cohort, we identified patients with AIS large vessel occlusion (LVO), with CT perfusion and RAPID (IschemaView) postprocessing as well as CT angiogram, and follow up MRI between November 2017 and June 2020. The effect of ASPECTS on 90-day disability outcomes was measured using logistic regression adjusted for age and NIHSS. To model the effect of strict ASPECTS cutoffs on EST eligibility for LVO patients, a regression discontinuity (RD) test was performed. In $\mathrm{RD}$ study design, observations lying closely on either side of the threshold are compared and an average treatment effect (of EST in this case) is measured.

Results Among the 173 patients that met inclusion criteria, median CTP-RAPID core volume was $5 \mathrm{~mL}$ [IQR:0-27], and median ASPECTS was 8 [IQR: 6-9]. Median NIHSS was 14 [IQR: 8-18], and 85\% were admitted with a baseline mRS of 0 . Sixty six percent of patients underwent EST, 32\% had final TICI of $2 \mathrm{~b}, 8 \% 2 \mathrm{c}$, and $60 \% 3.45 \%$ had a $90-\mathrm{mRS}$ of $0-2$. ASPECTS was associated with likelihood of functional independence at 90 days, with continuous increase in likelihood over the entire scale after adjusting for age and NIHSS (OR 1.09 [95\% CI 0.91 - 1.31], and figure panel A). In regression discontinuity analysis (figure Panel B), applying a strict threshold of ASPECTS $\geq 5$ to offer EST resulted in a notable discontinuity in clinical outcomes, demonstrating a substantial treatment effect of EST close to this threshold.

Conclusion ASPECT scores at presentation in patients with LVO were associated with 90-day disability outcomes in a 